\title{
NEW CHAINING CRITERIA IN DIPOLAR FLUIDS BASED ON MONTE CARLO SIMULATIONS
}

\author{
SÁNDOR NAGY*1,2 \\ ${ }^{1}$ Berzsenyi Dániel Teacher Training Centre, Eötvös Loránd University, Károlyi Gáspár tér 4. A-building, 9700 \\ Szombathely, HUNGARY \\ ${ }^{2}$ Institute of Mechatronics Engineering and Research, University of Pannonia, Gasparich Márk u. 18/A, \\ 8900 Zalaegerszeg, HUNGARY
}

\begin{abstract}
A new energy-based chaining criterion was introduced in dipolar systems based on an earlier article by the author, in which the probability of chaining for adjacent particles in a new formula of magnetic susceptibility was used. The probability of chaining and the magnitude of the energy criterion can be calculated from the Monte Carlo (MC) simulation values of magnetic susceptibility. The energy criterion also depends on the dipole moment and the density. At high densities, the energy criterion is well below $70-75 \%$. In addition, it was confirmed by simulation results that the chain length distribution follows a geometric distribution. How the probability of chaining depends on the energy criterion was given empirically and two parameters were fitted to it.
\end{abstract}

Keywords: dipolar fluids, chain formation, energy criterion

\section{Introduction}

According to the literature, the criterion of chaining in dipolar systems is unclear. Usually, an energy criterion is used to decide whether two adjacent particles form part of a chain. The energy criterion defines the limit at a certain level of interaction energy between them, which is usually $70-75 \%$ of the minimum pair interaction energy [1-4]. According to another definition, if the pair interaction energy is negative and the two particles are closer together than 1.3 in diameter unit, then chaining occurs $[5,6]$. The problem with this is that the minimum pair interaction energy depends on the magnitude of the dipole moment, so an identical amount of pair interaction energy between two adjacent particles indicates chaining in one case but not in the other. In another article, the author examined [7] the probability density function of pair interaction energies in a dipolar hard sphere (DHS) system and found that no unit jump-like change in the frequency of the pair interaction energy would justify the introduction of a general criterion based on the pair interaction energy. Furthermore, such a general definition does not take into account the effect of density, while it can be assumed that chaining occurs at different densities and different energy levels of the same dipole moments. Therefore, this study seeks to determine the magnitude of the energy criterion from another source, that is, a real, measurable, physical parameter. The physical parameter in this case is magnetic susceptibility.

In a previous article [8], the author stated that the chain length distribution in dipolar fluids follows a geometric distribution. If the probability that two adjacent particles form a chain is denoted by $p$, it can be deduced that the chain length distribution is

$$
g_{k}=(1-p) p^{k-1},
$$

where $k$ stands for the chain length. The particle size distribution, which yields the proportion of particles in exactly $k$-long chains is

$$
h_{k}=(1-p)^{2} k p^{k-1} .
$$

The average chain length is derived from the properties of the geometric distribution: $1 /(1-p)$. Assuming that a chain of length $k$ behaves as if its dipole moment is $k m$, its initial magnetic susceptibility can be deduced (in c.g.s. units) as

$$
\chi_{0}=\frac{1+p}{1-p} \chi_{\mathrm{L}}\left(1+\frac{4 \pi}{3} \chi_{\mathrm{L}}\right)
$$

where $\chi_{\mathrm{L}}$ denotes Langevin susceptibility [9],

$$
\chi_{\mathrm{L}}=\frac{\rho m^{2}}{3 k_{\mathrm{B}} T},
$$

$\rho$ stands for the density, $T$ represents the temperature, and $k_{\mathrm{B}}$ refers to the Boltzmann constant. In Eq. 3, the expression

$$
\chi_{\mathrm{P}}=\chi_{\mathrm{L}}\left(1+\frac{4 \pi}{3} \chi_{\mathrm{L}}\right)
$$

\footnotetext{
*Correspondence: sata123.sandor@gmail.com
} 
calculates the initial magnetic susceptibility from Pshenichnikov's theory [10]. Eq. 3 can be used to determine the value of $p$ at a given density and dipole moment, since $\chi_{0}$ can be determined from simulations and $\chi_{\mathrm{P}}$ can be calculated exactly from

$$
p=\frac{\chi_{0}-\chi_{\mathrm{P}}}{\chi_{0}+\chi_{\mathrm{P}}} .
$$

If the occurrence of chaining is linked to an energy criterion, it is clear that the value of $p$ depends on the magnitude of the energy criterion. Thus, if the correct value of $p$ is known from Eq. 6, the magnitude of the correct energy criterion can be obtained from the relationship $p-U_{\text {lim }}$. The structure of the results is as follows: firstly, through several examples, it is shown that for any energy criterion, the number of chains follows a geometric distribution; secondly, the $p$ values calculated from the magnetic susceptibility values are given; thirdly, from simulations, the $U_{\text {lim }}$ values are determined that provide the desired $p$ value at a given density and dipole moment; finally, by fitting, an empirical formula is derived to describe the relationship $p-U_{\text {lim }}$.

\section{Simulations and results}

Monte Carlo simulations of DHS fluids were performed using a canonical NVT ensemble. Boltzmann sampling [11], periodic boundary conditions and the minimumimage convention were applied. In order to take into account the long-range character of the dipolar interaction, the reaction field method under periodic boundary conditions of conduction was used. After 100, 000 equilibration periods, $1,000,000$ production cycles were conducted involving $N=1,000$ particles. The reduced density was calculated by $\rho^{*}=\rho \sigma^{3}$ and the reduced dipole moment by $m^{*}=m / \sqrt{\sigma^{3} k_{B} T}$, where $\sigma$ denotes the diameter of the particles. The pair interaction energy between two particles in a DHS system is determined only by the dipolar energy:

$$
U_{i j}^{\mathrm{dd}}=-\frac{m^{2}}{r_{i j}^{3}}\left[3\left(\widehat{\mathbf{m}}_{i} \cdot \widehat{\mathbf{r}}_{i j}\right)\left(\widehat{\mathbf{m}}_{j} \cdot \widehat{\mathbf{r}}_{i j}\right)-\left(\widehat{\mathbf{m}}_{i} \cdot \widehat{\mathbf{m}}_{j}\right)\right],
$$

where the particles have dipole moments of strength $m$ of an orientation given by unit vector $\widehat{\mathbf{m}}$, moreover, the distance between the centers of the particles is denoted by $r_{i j}$ and $\widehat{\mathbf{r}}_{i j}=\mathbf{r}_{i j} / r_{i j}$. The dots symbolize the scalar product. The lowest and most favorable energy value was determined by $U_{\text {min }}^{\mathrm{dd}}=-2 \mathrm{~m}^{2} / \sigma^{3}$. The magnitude of the energy criterion $(u)$ was given in the usual way in proportion to this: $u=U^{\mathrm{dd}} / U_{\min }^{\mathrm{dd}}$.

To determine the chain length distribution $\left(g_{k}\right)$, the number of chains of a given length was counted in each cycle. This required a predefined energy criterion. Rearranging Eq. 1 leads to the chain length distribution:

$$
\lg \left(g_{k}\right)=\lg (1-p)+(k-1) \lg (p) .
$$

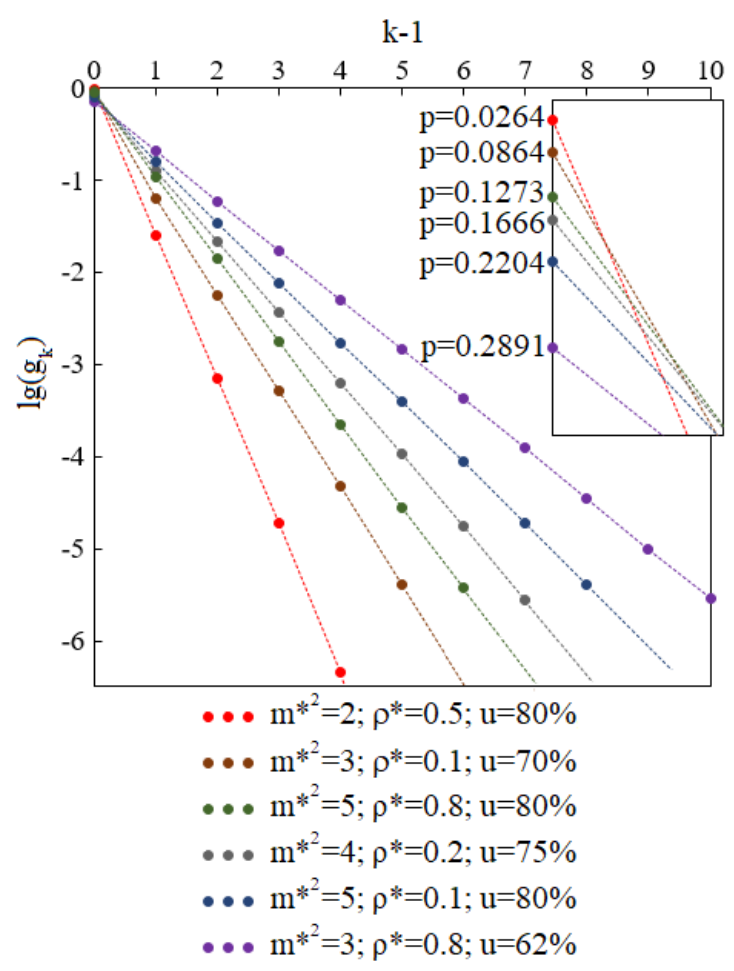

Figure 1: The logarithm of the chain length distribution as a function of chain length at six different dipole moments, densities and energy criteria. According to Eq. 8, the value of $p$ (probability of chaining) is also derived from the gradient of the fitted lines as well as the intercept of the vertical axis.

From this, it can be seen that if the logarithm of the chain length distribution is plotted as a function of $k-1$, the gradient of the fitted straight line yields the logarithm $p$, while the logarithm of the vertical intercept gives $1-p$. Since in each case the resulting lines are linear, it follows that the chain length distribution does indeed follow a geometric distribution. Fig. 1 shows the simulation results for the chain length distribution obtained for six different combinations of dipole moments, densities and energy criteria. It can be seen that the fitted lines are linear on the logarithmic scale in all cases. It is clear from the inset graphs that the larger its gradient, the closer its intercept is to zero. The $p$ values shown in Fig. 1 were derived from the gradient of the fitted lines.

Further results are summarized in Table 1. In the third column of Table 1, using the terms mentioned in the Introduction (Section 1), the values of $p$ are given. The values of $\chi_{\mathrm{P}}$ can be precisely calculated from Eq. 5. The values of $\chi_{0}$ are derived from the simulations. (For the simulation results of $\chi_{0}$, reference [12] was used. The missing $\chi_{0}$ data were supplemented with our own simulation results.)

This was followed by the determination of $u_{\mathrm{lim}}$, also from our own simulations. According to Eq. 2, the number of particles that do not form a chain or, in other words, which form single-element chains is $h_{1}=(1-p)^{2}$. Thus, in each step of the simulation, it was only nec- 
Table 1: The probabilities of chaining ( $3^{\text {rd }}$ column), the values of the energy criterion ( $4^{\text {th }}$ column) according to the simulations and the values of the fitted curves $\left(5^{\text {th }}\right.$ and $6^{\text {th }}$ columns) according to Eq. 9

\begin{tabular}{cccccc}
\hline$\left(m^{*}\right)^{2}$ & $\rho^{*}$ & $p$ & $u_{\text {lim }}$ & $A$ & $B$ \\
\hline 2 & 0.1 & 0.0135 & 0.74 & 0.203 & 2.902 \\
2 & 0.2 & 0.0209 & 0.76 & 0.358 & 2.864 \\
2 & 0.3 & 0.0316 & 0.75 & 0.492 & 2.863 \\
2 & 0.4 & 0.0231 & 0.80 & 0.623 & 2.881 \\
2 & 0.5 & 0.0254 & 0.80 & 0.751 & 2.893 \\
2 & 0.6 & 0.0387 & 0.78 & 0.885 & 2.901 \\
2 & 0.7 & 0.0523 & 0.77 & 1.022 & 2.895 \\
2 & 0.8 & 0.1674 & 0.66 & 1.167 & 2.874 \\
2 & 0.9 & 0.2641 & 0.62 & 1.307 & 2.821 \\
3 & 0.1 & 0.0802 & 0.68 & 0.426 & 2.284 \\
3 & 0.2 & 0.0951 & 0.71 & 0.607 & 2.291 \\
3 & 0.3 & 0.0772 & 0.75 & 0.732 & 2.333 \\
3 & 0.4 & 0.0721 & 0.77 & 0.839 & 2.388 \\
3 & 0.5 & 0.0762 & 0.77 & 0.943 & 2.442 \\
3 & 0.6 & 0.0840 & 0.76 & 1.048 & 2.498 \\
3 & 0.7 & 0.1889 & 0.67 & 1.154 & 2.535 \\
3 & 0.8 & 0.3788 & 0.57 & 1.270 & 2.560 \\
3 & 0.9 & 0.6745 & 0.45 & 1.383 & 2.547 \\
4 & 0.1 & 0.2183 & 0.67 & 0.761 & 1.801 \\
4 & 0.2 & 0.1777 & 0.73 & 0.900 & 1.842 \\
4 & 0.3 & 0.1278 & 0.77 & 0.975 & 1.900 \\
4 & 0.4 & 0.1174 & 0.78 & 1.044 & 1.989 \\
4 & 0.5 & 0.1169 & 0.78 & 1.107 & 2.069 \\
4 & 0.6 & 0.1818 & 0.72 & 1.180 & 2.159 \\
4 & 0.7 & 0.4016 & 0.59 & 1.258 & 2.237 \\
4 & 0.8 & 0.6814 & 0.46 & 1.341 & 2.295 \\
\hline & & & & &
\end{tabular}

essary to count how many particles have the minimum pair interaction energy (counted individually for the other particles) greater than the examined energy criterion. This greatly simplified the complexity of the simulations. Therefore, the value of $p$ belonging to the given energy criterion was determined, and for each dipole moment and density, a function was generated to create a relationship between $p$ and $u_{\mathrm{lim}}$. Of these, four are shown in Fig. 2 . The dashed lines indicate the $u_{\text {lim }}$ values of the already defined $p$ values of these functions.

The fourth column of Table 1 shows the $u_{\text {lim }}$ values for each dipole moment, density and value of $p$ which are also plotted in Fig. 3. (The uncertainty of the functions shown in Fig. 3 stems from the uncertainty of the magnetic susceptibilities. For dipole moments less than $\left(m^{*}\right)^{2}=2$, this new energy criterion cannot be examined precisely because the uncertainty in the magnetic susceptibility is too great.) It can be seen that the frequently mentioned $70-75 \%$ criterion is more or less valid, although it differs significantly from it at high densities. Interestingly, the energy criterion is higher at medium densities than at low densities. This may be because the

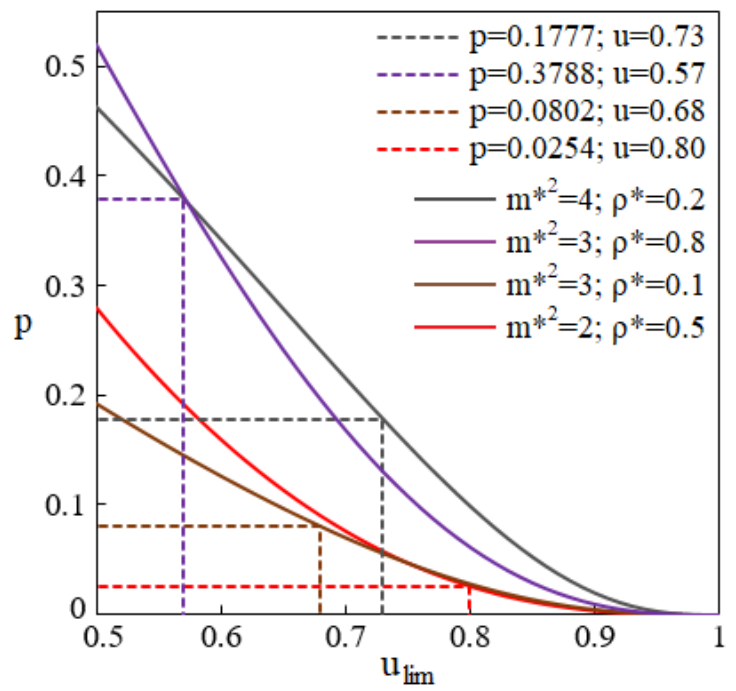

Figure 2: The probability of chaining as a function of the value of the energy criterion at four different dipole moments and densities. The dashed lines show the true values of $p$ and thus $u_{\mathrm{lim}}$ as well.

chains are so close to each other at medium densities that they have an effect on each other, but do not at low densities. It is true that at high densities this effect is even stronger, but at the same time, the strength of the forces acting on the chain also increases.

In the following, the relationships between the $p-$ $u_{\lim }$ functions are specified by fitting. Four of these are shown in Fig. 2. In Fig. 4, for each of the three dipole moments examined, these functions are plotted at two densities. Since the functions are close to zero around $u_{\lim }=1$, the cosine function seems to be a good choice for describing the curves as follows:

$$
p=A \cos ^{B}\left(\frac{\pi}{2} u_{\mathrm{lim}}\right)
$$



Figure 3: The values of $u_{\mathrm{lim}}$ as a function of the reduced density at three different dipole moments. 


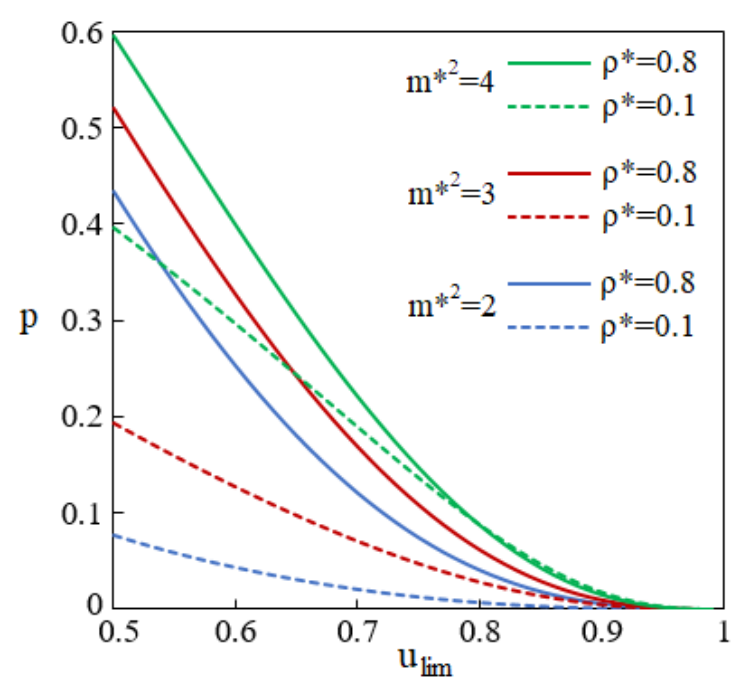

Figure 4: The probability of chaining as a function of the energy criterion at three different dipole moments. Dashed lines refer to low densities $\left(\rho^{*}=0.1\right)$, solid lines refer to high densities $\left(\rho^{*}=0.8\right)$. The lines of intermediate densities are between these two lines.

where $A$ and $B$ are constants and their magnitudes are given in the fifth and sixth columns of Table 1 . Fits were made within the range $u_{\text {lim }}=0.5-1$. The absolute error in the values of $p$ is not greater than 0.01285 in all the cases examined.

\section{Conclusion}

The main result of this article is shown in Fig. 3. A well-explained energy-based chaining criterion resulting from magnetic susceptibility was defined. From the simulated values of magnetic susceptibility, the probability of chaining was calculated. From this, the magnitude of the chaining criterion was derived using simulations as well. Therefore, a well-explained theory was developed to define the chaining energy criterion, which produced different results for different values of density and dipole moment in DHS systems. The criterion value of $70-75 \%$ commonly given in the literature is only approximately true at low densities $\left(\rho^{*} \leq 0.3\right)$. At medium densities $\left(0.3<\rho^{*}<0.6\right)$, the values are generally higher, while at high densities $\left(\rho^{*} \geq 0.6\right)$, they are much lower.

\section{Acknowledgement}

This research was supported by the European Union and co-financed by the European Social Fund under the project EFOP-3.6.2-16-2017-00002.

\section{REFERENCES}

[1] Ivanov, A. O.; Wang, Z.; Holm, C.: Applying the chain formation model to magnetic properties of aggregated ferrofluids, Phys. Rev. E, 2004, 69(3), 031206 DOI: 10.1103/PhysRevE.69.031206

[2] Wang, Z.; Holm, C.; Müller, H. W.: Molecular dynamics study on the equilibrium magnetization properties and structure of ferrofluids, Phys. Rev. E, 2002, 66(2), 021405 DOI: 10.1103/PhysRevE.66.021405

[3] Valiskó, M.; Varga, T.; Baczoni, A.; Boda, D.: The structure of strongly dipolar hard sphere fluids with extended dipoles by Monte Carlo simulations, Mol. Phys., 2010, 108(1), 87-96 DOI: 10.1080/00268970903514553

[4] Tavares, J. M.; Weis, J. J.; Telo da Gama, M. M.: Strongly dipolar fluids at low densities compared to living polymers, Phys. Rev. E, 1999, 59(4), 43884395 DOI: 10.1103/PhysRevE.59.4388

[5] Kantorovich, S.; Ivanov, A. O.; Rovigatti, L.; Tavares, J. M.; Sciortino, F.: Nonmonotonic magnetic susceptibility of dipolar hard-spheres at low temperature and density, Phys. Rev. Lett., 2013, 110(14), 148306 DOI: 10.1103/PhysRevLett.110.148306

[6] Rovigatti, L.; Russo, J.; Sciortino, F.: Structural properties of the dipolar hard-sphere fluid at low temperatures and densities, Soft Matt., 2012, 8(23), 6310-6319 DOI: $10.1039 / \mathrm{C} 2 \mathrm{SM} 25192 \mathrm{~B}$

[7] Nagy, S.: The frequency of the two lowest energies of interaction in dipolar hard sphere systems, Anal. Tech. Szeged., 2020, 14(2) in press

[8] Nagy, S.: The initial magnetic susceptibility of dense aggregated dipolar fluids, Hung. J. Ind. Chem., 2018, 46(2), 47-54 DOI: 10.1515/hjic-2018-0018

[9] Langevin, P.: Sur la théorie du magnétisme, J. Phys. Theor. Appl., 1905, 4(1), 678-693 DOI: 10.1051/jphystap:019050040067800

[10] Pshenichnikov, A. F.; Mekhonoshin, V. V.: Equilibrium magnetization and microstructure of the system of superparamagnetic interacting particles: numerical simulation, J. Magn. Magn. Mater., 2000, 213(3), 357-369 DOI: 10.1016/S0304-8853(99)00829-X

[11] Allen, M. P.; Tildesley, D. J.: Computer simulation of liquids (Clarendon Press, Oxford) 1987, ISBN: 978-0-198-55645-9

[12] Theiss, M.; Gross, J.: Dipolar hard spheres: comprehensive data from Monte Carlo simulations, J. Chem. Eng. Data, 2019, 64(2), 827-832 DOI: 10.1021/acs.jced.8b01169 